\title{
Continuous time evolution from iterated maps and Carleman linearization
}

\author{
P. Gralewicz and K. Kowalski \\ Department of Theoretical Physics, University of Łódź, ul. Pomorska 149/153, 90-236 tódź, Poland \\ Using the Carleman linearization technique the continuous iteration of a mapping \\ is studied. Based on the detailed analysis of the Carleman embedding matrix the \\ precise mathematical meaning is given to such notion. The ordinary differential \\ equations referring to continuous iterations are identified and the discussion of the \\ relationship between them and the corresponding iterated maps is performed.
}

\section{INTRODUCTION}

There are well known examples of how to relate a continuous time differential system

$$
\dot{x}(t)=F(x(t))
$$

where $F: \mathbf{R}^{k} \rightarrow \mathbf{R}^{k}$, to an iteration of a map of $\mathbf{R}^{s}$ into itself. We only recall the Euler's broken line method and the Poincaré map. An example of the inverse procedure to the discretization has been recently discussed in [1]. Namely, a precise meaning has been given therein to the notion of a "continuous iteration" of a mapping, that is a continuous counterpart of the iterates

$$
f^{n}(x)=f\left(f^{n-1}(x)\right)
$$

The purpose of this work is to introduce an alternative formalism for the study of the continuous iterations based on the classical Carleman linearization technique which is more general than the approach taken up in 远. In section II we briefly introduce the Carleman linearization technique. Section III is devoted to the detailed analysis of the properties of the Carleman embedding matrix which are crucial for the actual treatment. Based on the observations of section III we find in section IV an explicit formula on continuous iterations of a mapping and show its connection with a linearization transformation for a corresponding recurrence. The theory is illustrated by an example of the logistic equation. In section $\mathrm{V}$ we introduce the ordinary differential equation referring to the continuous iteration and find a simple relation between the Carleman embedding matrices corresponding to the continuous and discrete time cases. 


\section{THE CARLEMAN LINEARIZATION}

We begin by recalling the Carleman linearization technique [2]. Consider the system

$$
\dot{x}=F(x),
$$

where $F: \mathbf{R}^{k} \rightarrow \mathbf{R}^{k}$ and $F$ is analytic in $x$. Having in mind the applications of the Carleman technique in the study of the iterated one-dimensional maps discussed in this work we restrict for brevity to the case with $k=1$, i.e. the ordinary differential equation (2.1). On making the ansatz

$$
x_{j}:=x^{j}, \quad j=1,2, \ldots,
$$

where $x$ fulfils (2.1) we arrive at the infinite linear system

$$
\dot{x}_{j}=\sum_{k=0}^{\infty} L_{j k} x_{k}
$$

with the constant coefficient matrix $L_{j k}$. Clearly, in view of (2.2) the finite system (2.1) is embedded into the infinite system (2.3). Therefore, the Carleman linearization is also referred to as the Carleman embedding technique. Recently, the Carleman approach has been succesfully applied to the solution of numerous nonlinear problems (see [3] and references therein). We only recall the application of the Carleman linearization technique for calculating Lyapunov exponents [4] and finding first integrals for the Lorenz system [5].

As shown by Steeb [6] the Carleman embedding can be easily generalized to the case with nonlinear recurrences of the form

$$
x_{n+1}=f\left(x_{n}\right),
$$

where $f$ is analytic in $x_{n}$. Indeed, in analogy with (2.2) we set

$$
x_{j n}:=x_{n}^{j}
$$

where $x_{n}$ fulfils (2.4), which leads to the infinite-dimensional linear system of difference equations such that

$$
x_{j n+1}=\sum_{k=0}^{\infty} M_{j k} x_{k n} .
$$

As with the case of the ordinary differential equations the finite-dimensional recurrence (2.4) is embedded into the infinite linear system (2.5).

\section{THE CARLEMAN EMBEDDING MATRIX FOR NONLINEAR RECURRENCES}

In this section we study the properties of the Carleman embedding matrix for nonlinear recurrences specified by (2.6) which are utilized in the actual formalism. We now return to (2.6). Let 
$M(f)$ designate the Carleman matrix referring to the recurrence (2.4). Equations (2.4), (2.5) and (2.6) taken together yield

$$
(f(x))^{j}=\sum_{k=0}^{\infty} M_{j k}(f) x^{k}
$$

so

$$
M_{j k}(f)=\left.\frac{1}{k !} \frac{d^{k}(f(x))^{j}}{d x^{k}}\right|_{x=0} .
$$

Let

$$
f(x)=\sum_{k=0}^{\infty} f_{k} x^{k}
$$

The matrix $M(f)$ can be alternatively defined with the help of the coefficients of the expansion $(3.3)$ as

$$
M_{j k}(f)= \begin{cases}\delta_{0 k} & \text { for } j=0, \\ \sum_{m_{1}+m_{2}+\ldots+m_{j}=k} f_{m_{1}} f_{m_{2}} \cdots f_{m_{j}} & \text { for } j \geq 1 .\end{cases}
$$

The first few elements of the matrix $M(f)$ are

$$
M(f)=\left(\begin{array}{lllll}
1 & 0 & 0 & 0 & \cdots \\
f_{0} & f_{1} & f_{2} & f_{3} & \cdots \\
f_{0}^{2} & 2 f_{0} f_{1} & 2 f_{0} f_{2}+f_{1}^{2} & 2\left(f_{0} f_{3}+f_{1} f_{2}\right) & \ldots \\
f_{0}^{3} & 3 f_{0}^{2} f_{1} & 3\left(f_{0}^{2} f_{2}+f_{0} f_{1}^{2}\right) & 3 f_{0}^{2} f_{3}+6 f_{0} f_{1} f_{2}+f_{1}^{3} & \cdots \\
\vdots & \vdots & \vdots & \vdots &
\end{array}\right) .
$$

We remark that usage of the formula (3.2) or (3.4) is not the most effective way of calculating the elements of the matrix $M(f)$. The simpler possibility is to apply the relation

$$
M_{j k}(f)=\frac{1}{2 \pi} \int_{0}^{2 \pi} e^{\mathrm{i} k \varphi}\left(f\left(e^{-\mathrm{i} \varphi}\right)\right)^{j} d \varphi
$$

following directly from (3.1) and the well-known fact that the functions of the form $e^{\mathrm{in \varphi}}$, where $n=0,1,2, \ldots$, form the orthonormal basis of the space of the square integrable functions on a unit circle.

Example: Consider the logistic equation

$$
x_{n+1}=\mu x_{n}\left(1-x_{n}\right) .
$$

Using the relation (3.6) we easily obtain the following formula on the elements of the corresponding Carleman matrix $M$ : 


$$
M_{j k}=(-1)^{k-j}\left(\begin{array}{c}
j \\
k-j
\end{array}\right) \mu^{j}
$$

(the vanishing of $M_{j k}$ in the case with $2 j<k$ is understood).

We now focus our attention on the mapping

$$
f \rightarrow M(f)
$$

A remarkable property of (3.9) is that it provides a representation of a semigroup of analytic functions with multiplication defined as the composition operation, that is

$$
M(f \circ g)=M(f) M(g)
$$

The relation (3.10) is an immediate consequence of (3.1). Clearly,

$$
M(\mathrm{id})=I
$$

where $\operatorname{id}(x) \equiv x$ is an identity function playing the role of a neutral element for the semigroup of the analytic functions, $I$ is the identity matrix, and whenever exists the inverse $f^{-1}$ of $f$, then

$$
M\left(f^{-1}\right)=M^{-1}(f)
$$

Finally, it is clear in view of (3.10) that iterations $f^{n}$ of a function $f$ are represented by matrix powers, i.e.

$$
M\left(f^{n}\right)=M^{n}(f)
$$

Having in mind the form of the relation (3.13) it is plausible to define the continuous iterations $f^{t}$ of $f$, where $t$ is a real parameter, by

$$
M\left(f^{t}\right)=M^{t}(f)
$$

Thus, the problem of the precise definition of continuous iterations can be reduced to finding the powers $M^{t}$ of the matrix $M$ for a non-integer $t$.

We recall that the infinite-dimensional (anti)representations of the formal power series were originally studied in [1]. The counterpart of the formula (3.10) introduced therein describing the anti-representation is of the form

$$
B(f \circ g)=B(g) B(f),
$$

where $B(f)$ are the Bell matrices specified by

$$
B_{j k}(f)=\left.\frac{1}{k !} \frac{d^{j}(f(x))^{k}}{d x^{k}}\right|_{x=0}, \quad j, k=1,2, \ldots,
$$


and it is assumed that the function $f$ given by the formal power series satisfies $f(0)=0$. We point out that such assumption is rather restrictive one and it is not satisfied in such important cases as for example $f(z)=z^{2}+c$, related to the celebrated Mandelbrot fractal. Evidently, we have

$$
B\left(f^{n}\right)=B^{n}(f) .
$$

As with (3.13) the relation (3.17) was the point of departure in [1] to define the continuous iterations. Nevertheless, the approach taken up therein is less general and it seems to be more complicated than that introduced in the next section of this work. We finally remark that in opposition to the actual treatment there is no interpretation of the Bell matrices $B(f)$ provided in 11] connected with an infinite-dimensional linearization of the original nonlinear recurrence (2.4).

\section{POWERS OF THE CARLEMAN EMBEDDING MATRICES AND CONTINUOUS ITERATIONS}

As mentioned in the previous section (see formula (3.14)) the problem of the precise definition of continuous iterations reduces to finding the non-integer powers $M^{t}$ of the matrix $M(f)$. Our purpose now is to discuss this point in a more detail. We first observe that the problem under investigation can be furthermore cast into determining the transformation diagonalizing $M$ such that

$$
M(f)=U^{-1} \Lambda U
$$

where $\Lambda$ is diagonal, i.e. $\Lambda_{j k}=\lambda_{j} \delta_{j k}$. In fact, (4.1) leads to the following formula on the powers of the matrix $M$ :

$$
M^{t}(f)=U^{-1} \Lambda^{t} U
$$

where $\left(\Lambda^{t}\right)_{j k}=\lambda_{j}^{t} \delta_{j k}$.

In order to diagonalize the matrix $M$ we first bring it down to the triangular form. Consider (2.4). Let us assume that $x_{n}=x_{*}$ is a stationary solution to (2.4), that is $x_{*}$ is a fixed point of $f$ such that

$$
f\left(x_{*}\right)=x_{*} .
$$

As with ordinary differential equations we can switch over to new variables

$$
x_{n}^{\prime}=x_{n}-x_{*},
$$

so that the resulting nonlinear recurrence

$$
x_{n+1}^{\prime}=g\left(x_{n}^{\prime}\right) \text {, }
$$


where $g\left(x_{n}^{\prime}\right)=f\left(x_{n}^{\prime}+x_{*}\right)-x_{*}$, obeys

$$
g(0)=0
$$

Using the definition (3.4) one can easily check that the condition (4.6) leads to the upper triangular Carleman embedding matrix $M(g)$. Further, we have

$$
g=h \circ f \circ h^{-1}
$$

where

$$
h(x)=x-x_{*} .
$$

Using (3.10), (4.7) and (3.2) we arrive at the matrix relation of the form

$$
M(g)=T_{x_{*}} M(f) T_{x_{*}}^{-1},
$$

where $T_{x_{*}}=M(h)$, and

$$
\left(T_{x_{*}}\right)_{i j}= \begin{cases}\left(\begin{array}{l}
j \\
k
\end{array}\right)\left(-x_{*}\right)^{j-k} & \text { for } j \geq k \\
0 & \text { for } j<k\end{cases}
$$

Evidently,

$$
\left(T_{x_{*}}^{-1}\right)_{i j}= \begin{cases}\left(\begin{array}{l}
j \\
k
\end{array}\right) x_{*}^{j-k} & \text { for } j \geq k \\
0 & \text { for } j<k\end{cases}
$$

We have thus shown that the Carleman embedding matrix $M(f)$ corresponding to (2.4), where $f$ fulfils (4.3) can be reduced by means of the transformation (4.4) to the upper triangular form $M(g)$.

Now let

$$
g(x)=\sum_{k=0}^{\infty} g_{k} x^{k} .
$$

Taking into account (3.4) and (4.12) we find that the diagonal elements of the matrix $M(g)$ are

$$
M_{i i}(g)=g_{1}^{i}=\left(\frac{d g(0)}{d x}\right)^{i}=\left(\frac{d f\left(x_{*}\right)}{d x}\right)^{i}, \quad i=0,1,2, \ldots
$$

These elements coincide with the eigenvalues of the matrix $M(g)$ specified by [7]

$$
\psi M(g)=\lambda \psi
$$

where $\psi$ is an infinite row-vector. Suppose now that the eigenvalues $\lambda_{i}=M_{i i}(g)$ of the matrix $M(g)$ are mutually different. Since $\lambda_{i}=g_{1}^{i} \equiv \lambda^{i}$, therefore we then have the restrictive conditions $\lambda \neq 0$ and $\lambda \neq \sqrt[n]{1}$. It is easy to verify that the transformation diagonalizing $M(g)$ of the form 


$$
M(g)=V^{-1} \Lambda V
$$

where $\Lambda$ is diagonal, is given by the following recursive relations:

$$
\begin{array}{r}
V_{j k}= \begin{cases}\left(\lambda^{j}-\lambda^{k}\right)^{-1} \sum_{l=j}^{k-1} V_{j l} M_{l k}(g) & \text { for } j<k, \\
1 & \text { for } j=k, \\
0 & \text { for } j>k,\end{cases} \\
V_{j k}^{-1}= \begin{cases}\left(\lambda^{j}-\lambda^{k}\right)^{-1} \sum_{l=j+1}^{k} V_{l k}^{-1} M_{j l}(g) & \text { for } j<k, \\
1 & \text { for } j=k, \\
0 & \text { for } j>k,\end{cases}
\end{array}
$$

and

$$
\Lambda_{j k}=\lambda^{j} \delta_{j k}
$$

Finally, combining (4.15) and (4.9) we find that the transformation diagonalizing $M(f)$ can be expressed by (4.1), with

$$
U=V T_{x_{*}}
$$

We are now in a position to define the desired continuous iteration $f^{t}$. Indeed, eqs. (3.14), (3.1) and (4.1) taken together yield the following formula on $f^{t}$ :

$$
\begin{aligned}
f^{t}(x) & =\sum_{k=0}^{\infty}\left(M^{t}\right)_{1 k}(f) x^{k}=\sum_{k=0}^{\infty}\left(U^{-1} \Lambda^{t} U\right)_{1 k} x^{k} \\
& =\sum_{j k l m}\left(T_{x_{*}}^{-1}\right)_{1 j} V_{j k}^{-1} \lambda^{k t} V_{k l}\left(T_{x_{*}}\right)_{l m} x^{m} .
\end{aligned}
$$

On introducing the functions $\varphi_{k}(x)$ such that

$$
\varphi_{k}(x):= \begin{cases}\sum_{l=0}^{\infty} V_{1 k}^{-1} V_{k l}\left(x-x_{*}\right)^{l} & \text { for } k>0, \\ x_{*} & \text { for } k=0,\end{cases}
$$

we can write (4.20) in a more compact form. It follows that

$$
f^{t}(x)=\sum_{k=0}^{\infty} \lambda^{k t} \varphi_{k}(x) .
$$

Thus it turns out that the problem of the definition of continuous iterations can be brought down to the solution of the eigenvalue equation (4.14). Further, it is straightforward to show that the matrix $V$ satisfying (4.15) satisfies the relation (3.4). Using this we find that the matrix $V$ can be expressed by the solution to the eigenvalue equation (4.14) with the help of the following relation: 


$$
V_{j k}=\sum_{l=0}^{k} V_{j-1 l} \psi_{k-l},
$$

where $\psi_{k}$ are the coordinates of the vector $\psi$. Now it is not difficult to check that besides of the matrix $V$ also the diagonal matrix $\Lambda$ specified by (4.18) fulfils the relation (3.4). On passing with the use of (4.1) and (4.19) from matrices to functions we arrive at the following functional equation:

$$
f(x)=u^{-1}(\lambda u(x))
$$

where $u=v \circ h$, and $V=M(v)$. Clearly, the functional equivalent of (4.2) is of the form

$$
f^{t}(x)=u^{-1}\left(\lambda^{t} u(x)\right)
$$

It thus appears that the problem of the definition of the continuous iterations can be alternatively cast into the solution of the functional equation

$$
u(f(x))=\lambda u(x)
$$

The equation (4.26) is known in the literature. For example in [8] it was used for finding explicit solutions to nonlinear recurrences (2.4). We recall that the solution $u(x)$ of (4.26) is simply the linearization transformation

$$
x_{n}^{\prime}=u\left(x_{n}\right)
$$

reducing the solution of the nonlinear recurrence (2.4) to the linear one

$$
x_{n+1}^{\prime}=\lambda x_{n}^{\prime} .
$$

We point out that the connection of the functional equation (4.26) with an infinite-dimensional eigenvalue problem was found for the first time by the second author [9, 10] in the context of the Hilbert space description of nonlinear recurrences (2.4). The formula on continuous iterations analogous to (4.22) in the particular case of $f(0)=0$ was originally obtained in [1] with the use of the finite-dimensional truncations of the Bell matrices. We finally remark that our experience indicates that the recursive relation (4.23) on the matrix $V$ is of practical importance for the numerical solution of the functional equation (4.26).

Example: Consider as an illustrative example the well-known exactly solvable case of the logistic equation (3.7) with $\mu=4$

$$
x_{n+1}=4 x_{n}\left(1-x_{n}\right) .
$$

Using the identity 


$$
\arccos \left(2 x^{2}-1\right)=2 \arccos x, \quad 0 \leq x \leq 1,
$$

we get the solution to the functional equation (4.26), where $f(x)=4 x(1-x)$, of the form

$$
\begin{aligned}
\lambda & =4, \\
u(x) & =\frac{1}{4}[\arccos (1-2 x)]^{2} .
\end{aligned}
$$

Finally, taking into account (4.25) we get the desired formula on the continuous iterations such that

$$
f^{t}(x)=\frac{1}{2}\left\{1-\cos \left[2^{t} \arccos (1-2 x)\right]\right\}
$$

Referring back to (4.22) we find

$$
x_{*}=0, \quad h(x)=x, \quad \varphi_{0}=0, \quad \varphi_{k}(x)=\frac{1}{2}(-1)^{k+1} \frac{[\arccos (1-2 x)]^{2 k}}{(2 k !)}, \quad k \geq 1 .
$$

Thus the well-known solution to (4.29) of the form $x_{n}\left(x_{0}\right)=f^{n}\left(x_{0}\right)$, where $f^{n}\left(x_{0}\right)$ is given by (4.32) corresponds to the fixed point $x_{*}=0$ of $f(x)$. We now discuss the solution corresponding to the second fixed point $x_{*}=\frac{3}{4}$ of $f(x)$. On utilizing the identity

$$
2 \pi-\arccos \left(2 x^{2}-1\right)=2 \arccos x, \quad-1 \leq x<0,
$$

we arrive at the solution to (4.26), where

$$
\begin{aligned}
\lambda & =-2, \\
u(x) & =\frac{1}{2} \arccos (1-2 x)-\frac{\pi}{3} .
\end{aligned}
$$

Taking into account (4.25) and (4.35) we obtain

$$
f^{t}(x)=\frac{1}{2}\left\{1-\cos \left\{(-2)^{t}\left[\arccos (1-2 x)-\frac{2 \pi}{3}\right]+\frac{2 \pi}{3}\right\}\right\} .
$$

It can be checked with the use of (4.22), where $x_{*}=\frac{3}{4}$ and $h(x)=x-\frac{3}{4}$, that the obtained solution really refers to the fixed point $x_{*}=\frac{3}{4}$. Furthermore, a straightforward calculation shows that the function (4.32) is equivalent to (4.36) for non-negative integer $t$, that is the solutions of the logistic equation (4.29) corresponding to (4.32) and (4.36), respectively, such that $x_{n}\left(x_{0}\right)=f^{n}\left(x_{0}\right)$, coincide. Clearly, (4.32) is different from (4.36) for non-integer $t$. We conclude that in the case with the logistic equation (4.29) the continuous iteration is not unique. It should be noted that such ambiguity was not recognized in [1]. In fact, the solution (4.36) cannot be obtained by means of the approach introduced in [1]. We also remark that uniqueness of the continuous iterations referring to (4.29) is violated by the existence of the multiple equilibria for (4.29). It is suggested that it is the case for the general recurrence (2.4). We finally point out that due to the term $(-2)^{t}$ the function $f^{t}(x)$ given by (4.36) is complex-valued. 


\section{FROM ITERATED MAPS TO CONTINUOUS TIME EVOLUTION}

In the previous section we have investigated the continuous iterations $f^{t}(x)$ referring to the recurrence (2.4). Since we can interpret the continuous parameter $t$ as a "time variable", therefore the question naturally arises on the dynamics of $f^{t}(x)$. Consider the continuous iterations $f^{t}(x)$ and the corresponding powers of the Carleman embedding matrix $M^{t}(f)$. We have

$$
\begin{aligned}
\left(f^{t}(x)\right)^{j} & =\sum_{k=0}^{\infty}\left(M^{t}\right)_{j k} x^{k}, \\
\frac{d}{d t} M^{t} & =\ln M M^{t} .
\end{aligned}
$$

Eqs. (5.1) and (5.2) taken together yield

$$
\frac{d}{d t} f^{t}(x)=G\left(f^{t}(x)\right)
$$

where

$$
G(x)=\sum_{k=0}^{\infty}(\ln M)_{1 k} x^{k}
$$

We have thus shown that the continuous iterations $f^{t}(x)$ satisfy the ordinary differential equation (5.3) subject to the initial condition $f^{0}(x)=x$, i.e. $f^{t}$ is the flow corresponding to (5.3). Of course, since $f^{t}(x)$ is a flow therefore the vector field $G$ can be expressed by $f^{t}(x)$ with the help of the well-known relation

$$
G(x)=\left.\frac{d f^{t}(x)}{d t}\right|_{t=0} .
$$

An alternative form of the vector field $G$ can be obtained directly from (4.25). Indeed, differentiating both sides of (4.25) with respect to time we get (5.3) with

$$
G(x)=\ln \lambda \frac{d u^{-1}(u(x))}{d x} u(x) .
$$

Interestingly, there exists a remarkably simple relation between the Carleman linearization of the differential equation (5.3) and the Carleman linearization of the original recurrence (2.4). In fact, on setting

$$
x_{j}(t):=\left(f^{t}(x)\right)^{j}
$$

and using (3.1), (3.14) and (5.2) we arrive at the infinite linear system

$$
\dot{x}_{j}=\sum_{k=0}^{\infty} L_{j k} x_{k},
$$

where

$$
L=\ln M .
$$


Thus the logarithm of the Carleman embedding matrix related to the linearization of the recurrence (2.4) is simply the Carleman embedding matrix describing the linearization of the differential equation (5.3). We finally remark that whenever the solution $f^{n}(x)$ to (2.4) is chaotic then it is plausible to expect that the solution $f^{t}(x)$ of the differential equation (5.3) is also chaotic. As is well-known, the definition of chaotic systems presents a delicate problem, nevertheless it seems incredible that the one-dimensional autonomous system (5.3) would exhibit in any sense the chaotic behavior. The following example provides a possible solution of the problem.

Example: Consider the continuous iteration (4.32) corresponding to the solution of the logistic equation (4.29). An immediate consequence of (5.5) or (5.6) and (4.31) is the following differential equation satisfied by $f^{t}(x)$ :

$$
\frac{d f^{t}(x)}{d t}=\frac{1}{2} \ln 2 \sin \left\{\arccos \left[1-2 f^{t}(x)\right]\right\} \arccos \left[1-2 f^{t}(x)\right] .
$$

Notice that the principal part $\arccos (x)$ of the inverse cosine obeys $0 \leq \arccos (x) \leq \pi$, therefore the right-hand side of (5.10), that is $\frac{d f^{t}(x)}{d t}$, is non-negative. On the contrary, in view of (4.32) $f^{t}(x)$ oscillates. We conclude that $f^{t}(x)$ cannot satisfy (5.10) for arbitrary $t$. Indeed, an easy inspection shows that $f^{t}(x)$ satisfies the following differential equation:

$$
\frac{d f^{t}(x)}{d t}=\widetilde{G}\left(t, x, f^{t}(x)\right), \quad f^{0}(x)=x, \quad 0 \leq x \leq 1,
$$

where

$$
\begin{aligned}
& \widetilde{G}\left(t, x, f^{t}(x)\right)
\end{aligned}
$$

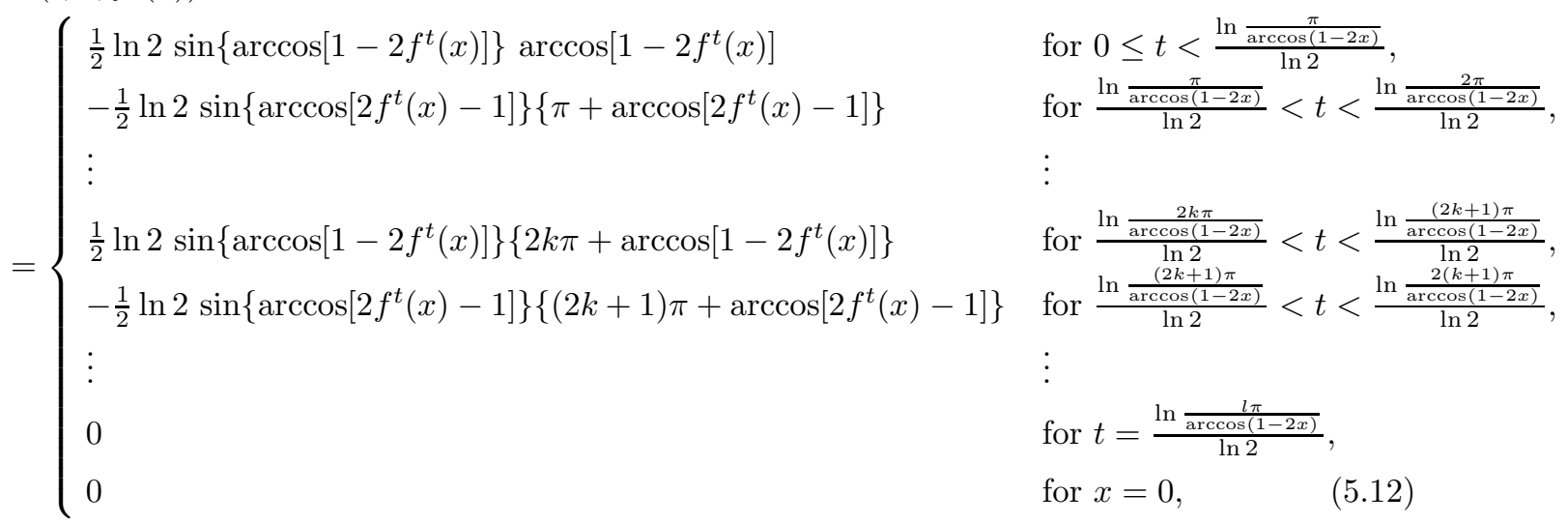

where $k, l=1,2, \ldots$. Thus, it turns out that (5.10) holds only for $0 \leq t<\frac{\ln \frac{\pi}{\arccos (1-2 x)}}{\ln 2}$. Nevertheless, in view of (5.12) the form of $\widetilde{G}$ is determined completely by (5.10). In fact, the continuation of (5.10) expressed by (5.12) is evidently implied by (5.10) and the fact that the inverse cosine is the infinitely-many-valued function. It seems that the adequate denomination for (5.10) would be the "principal part" of (5.11). As with (4.29) the differential equation (5.11) is also chaotic. In fact, by (4.32) $0 \leq f^{t}(x) \leq 1$, and the Lyapunov exponent $\sigma$ is 


$$
\sigma=\lim _{t \rightarrow \infty} \frac{1}{t} \ln \left|\frac{\partial f^{t}(x)}{\partial x}\right|=\ln 2 .
$$

As expected, this exponent coincides with the Lyapunov exponent for (4.29) [11]. Notice that the chaoticity of (5.11) does not contradict the well-known fact based on the Poincaré-Bendixon theorem that the minimal dimension of the phase space of the chaotic autonomous system is three. As a matter of fact the nonautonomous equation (5.11) refers to the two-dimensional phase space with coordinates $f^{t}$ and $t$. Nevertheless, becouse of the non-periodic dependence of the vector field $\widetilde{G}$ on the time variable $t$, the volume of the phase space occupied by the trajectories of the autonomous system corresponding to (5.11) is, in contrast to the assumption of the PoincaréBendixon theorem, infinite one.

\section{CONCLUSION}

In this work it is shown that the Carleman linearization of nonlinear recurrences defines the matrix representation of analytic functions. Such representation enables a sound definition of continuous iterations. On introducing the infinite-dimensional eigenvalue equation (4.14) related to the problem of the definition of continuous iterations and translating it back into the language of the composition of functions, we have arrived at the functional equivalent (4.26) of (4.14). As we have mentioned earlier that equation can be met in the literature. Nevertheless its application in the context of continuous iterations as with (4.25) is most probably new. In opposition to the alternative approach introduced by Aldrovandi and Freitas [1] the interpretation is provided in the actual treatment of the matrices representing functions in terms of the infinite dimensional linearization of the original nonlinear recurrence. We have also identified the (finite) dynamical system corresponding to continuous iterations, and we have found a simple formula (5.9) relating the Carleman embedding matrices in the discrete and continuous time cases. We note that the naive approach to the continuous iterations corresponding to (2.4) relying on the formal replacement of the discrete variable $n$ by the continuous one $t$ suggests only the delayed equation $x(t+1)=f(x(t))$ which is equivalent to the infinite dimensional system of ordinary differential equations. The simplicity of the approach taken up herein suggests that it would be a useful tool in the study of nonlinear recurrences as well as their continuous counterparts.

[1] R. Aldrovandi and L.P. Freitas, J. Math. Phys. 39, 5324 (1998).

[2] T. Carleman, Acta Mathematica 59, 63 (1932). 
[3] K. Kowalski and W.-H. Steeb, Nonlinear Dynamical Systems and Carleman Linearization (World Scientific, Singapore, 1991).

[4] R.F.S. Andrade, J. Math. Phys. 23, 2271 (1982).

[5] M. Kuś, J. Phys. A: Math. Gen. 16, L689 (1983).

[6] W.H. Steeb, Linearization procedure and nonlinear systems of differential and difference equations, in: Nonlinear Phenomena in Chemical Dynamics, eds. C. Vidal and A. Pacault (Springer, Berlin, 1981) p. 275 .

[7] R.A. Horn and C.R. Jackson, Matrix Analysis (Cambridge University Press, Cambridge, 1986).

[8] S. Katsura and W. Fukuda, Physica 130A, 597 (1985).

[9] K. Kowalski, Physica 195A, 137 (1993).

[10] K. Kowalski, Methods of Hilbert Spaces in the Theory of Nonlinear Dynamical Systems (World Scientific, Singapore, 1994).

[11] A.J. Lichtenberg and M.A. Lieberman, Regular and Stochastic Motion (Springer, New York, 1983). 